\title{
Flows on Discrete Traffic Flower
}

\author{
Alexander P. Buslaev ${ }^{1}$ \& Alexander G. Tatashev ${ }^{2}$ \\ ${ }^{1}$ Moscow Automobile and Road State Technical University, Moscow, Russia \\ ${ }^{2}$ Moscow Technical University of Communications and Informatics, Moscow, Russia \\ Correspondence: Alexander P. Buslaev, Moscow Automobile and Road State Technical University, Moscow, Russia.E- \\ mail: apal2006@yandex.ru
}

Received: November 9, 2016 Accepted: December 20, 2016 Online Published: January 23, 2016

doi:10.5539/jmr.v9n1p98 URL: http://dx.doi.org/10.5539/jmr.v9n1p98

Abstract A discrete dynamical system is considered in this paper. There are $L$ contours, which have a common point. There are $N_{i}$ cells and $M_{i}$ particles located in cells. Each particle moves on its contour in accordance with a given rule. Velocities of particles and other characteristics of the system are investigated.

Keywords: discrete dynamical systems, traffic models, Markov process, particles flow velocity, self-organization

\section{Introduction}

A dynamical system is considered in the paper. The system contains a finite set of cells. Particles are located in cells. Contours (cycles) are elementary supporters. A contour is a closed nonself-intersecting sequence of cells, which determines the direction of particles movement. A cell is a place of location of a particle. A particle is a unit of mass. The supporter is an oriented graph. Cells are vertices of the graph. An arc (oriented edge) connects two vertices if particles can move from one of these vertices to the other vertex. There is just one common point of contours. Contours have either just one common cell (node) or just one common point located between neighboring cells (alternating node). A feature of the considered system is that there is only one common point of contours. This supporter is called a flower. The traffic model with "flower" supporter has been introduced in (Buslaev, \& Yashina, 2009), (Buslaev, 2010), where an infinitesimal version of the system was considered.

A graph with alternating node is considered in the present paper, Figure 1. The common node is located between some pairs of cells on contours. In Figure 1, the node is shown as a square, and cells are shown as circles. The graph of the considered system is a bouquet graph. Therefore the considered system is called BAN (Bouquet Alternance Node). We discuss in what way the obtained results change in the case of a flower with the common cell if this cell is joined with the node.

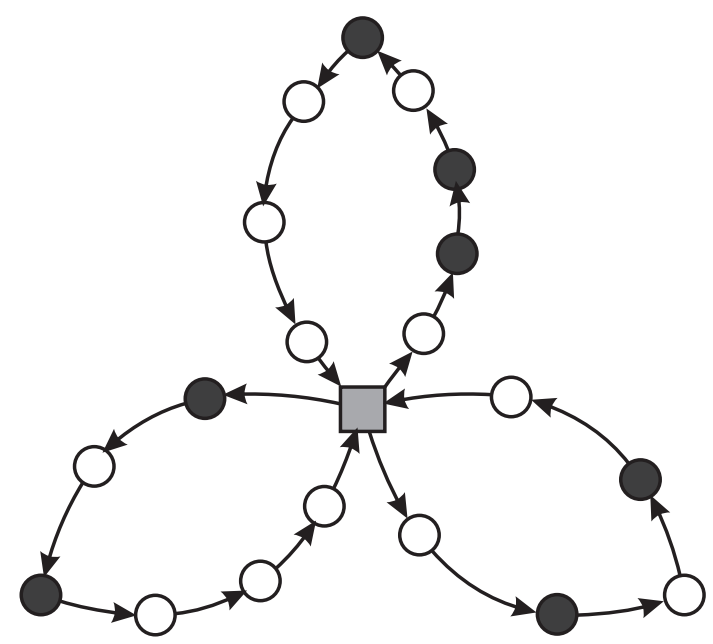

Figure 1. Bouquet with alternating node

Two types of traffic participants are considered. These types are individual movement and total-connected movement. In the case of individual movement, the particle shall be at the next time moment $(T+1)$ in the next cell if the next cell is vacant at the moment $T$. In the case of total-connected movement, Figure 2, the particle will be at the next time moment $(T+1)$ in the next cell if the next cell is vacant at the moment $T+1$. Hence, in the case of the total-connected movement the particle comes to the next cell at the next moment if this cell is released. In the case of total-connected 
movement neighboring particles form clusters. A cluster is the maximum subset of particles without vacant cells between these particles. Cluster moves synchronously if the next cell in the direction of movement is vacant. In Figure 2, cells are shown as circles, and the node is shown as rectangle. Occupied cells are highlighted in black. Dotted outlines show clusters.

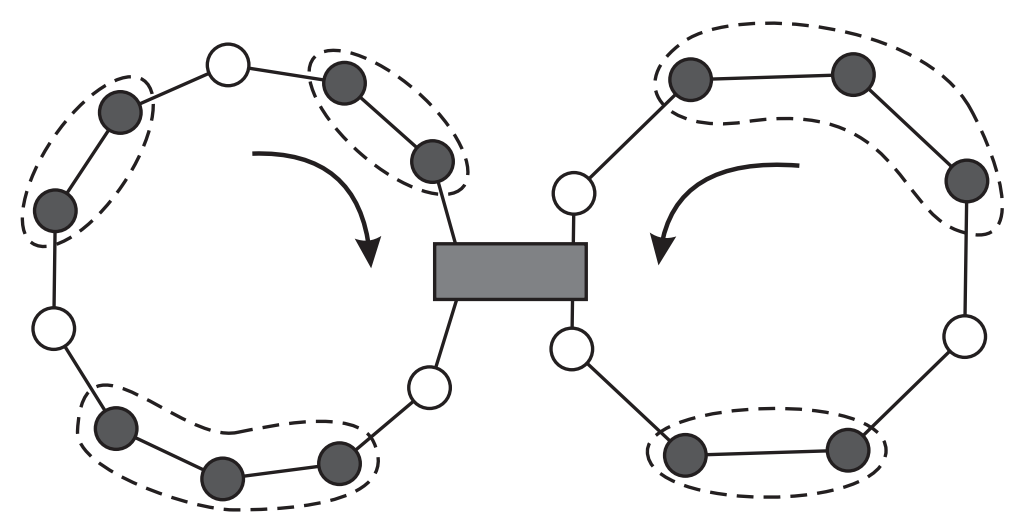

Figure 2. Total-connected movement

More than one cluster cannot move through the node simultaneously. The FIFO (First Input First Output) discipline acts at the node. Consider a cluster which has come to the node first. In accordance with the FIFO discipline, this cluster moves through the node first when the node releases. If leaders of some clusters come to the node simultaneously, then one of these clusters moves in accordance with the given conflict resolution rule. For example each of these clusters is chosen equiprobably (the egalitarian rule).

A traffic model was introduced in (Nagel, \& Schreckenberg, 1992). In this model, individual movement of a finite set of particles on a closed contour takes place. A hypothesis has been formulated in (Schadschneider, \& Schreckenberg, 1993). This hypothesis gives a formula that determines dependence of the particles velocity on the particles flow density. This formula has been proved in (Blank, 2000). The limit version of the model, considered in the present paper, is the continuous cluster model, considered in (Buslaev, \& Yashina, 2009), (Buslaev, 2010) (incompressible clusters). Discrete traffic models with total-connected movement were considered in (Bugaev, Buslaev, Kozlov, Tatashev, \& Yashina, 2013). In the case of the stochastic conflict resolution rule, considered in the present paper, the behavior of the system is stochastic. However, in present paper, we show that it is possible that the system behavior can come, after time with a finite expectation, to the state such that there will be no delays of particles movement in future. So, if the total number of particles is not greater than a critical number, then there are no conflicts after a finite moment. We say that the synergy state (self-organization) takes place. If the behavior of the system becomes deterministic, then the same sequence of states repeats periodically. These states form an orbit (in terms of dynamical systems theory (Halmos, 1956)), or a communicating class of states (in terms of Markov processes theory, (Feller, 1970), (Kemeny, \& Snell, 1976), (Borovkov, 1986)). This self-organization is also characteristic for dynamical systems with regular periodic structures, which have been introduced and investigated in (Kozlov, Buslaev, \& Tatashev, 2014), (Kozlov, Buslaev, Tatashev, \& Yashina, 2014), (Kozlov, Buslaev, \& Tatashev, 2015), (Kozlov, Buslaev, Tatashev, \& Yashina, 2015).

Analysis of the system is related to the theory of linear diophantine equations with two variables, (Buchstab, 1972). If there are no solutions of the appropriate diophantine equation, then there will be no conflict of a considered pair of particle if no other conflicts take place. If there is a solution of the equation, then a conflict will be in future. Thus analysis of diophantine equations gives a criterion of synergy.

The system with individual movement is considered in Section 2. We formulate the necessary and sufficient condition for a state of the system to be a state of synergy. We formulate necessary conditions and sufficient conditions for a state of synergy to exist.

Individual movement of particles on BAN with contours of the same length is considered in Section 3. We find the necessary and sufficient condition for a state of synergy to exist.

The system with total-connected movement is investigated in Sections 4 and 5. We have considered the system with two contours of the same length and a single cluster on each contour. We have found a necessary and sufficient condition for this system to come to the state of synergy after a finite time. We describe an approach to calculation of particles velocities on contours of this system. In the case of BAN with contours of the same length and any number of clusters, finally statements have been obtained. 


\section{Individual Movement on BAN}

\subsection{Formulation of Problem}

We consider a system (BAN) with $L$ contours. There are $N_{i}$ cells $(i, 0), \ldots,\left(i, N_{i}-1\right)$ and $M_{i}$ particles on the contour $i$, $M_{i}<N_{i}, i=1, \ldots, L$. Each particle is in one of the cells at any time moment $T=0,1,2, \ldots$ No more than one particle is in the cell. There is a common alternating node. The node is located such that a particle of the $i$ th contour moves through the node when this particle moves from the cell $(i, 0)$ to the cell $\left(i, N_{i}-1\right), i=1, \ldots, L$. If a particle of the contour $i$ is in the cell $(i, j)$ at moment $T$, the cell $(i, j-1)$ (substraction modulo $\left.N_{i}\right)$ is vacant, and the node is not located between cells $(i, j),(i, j-1)$ or no particle attempts to move through the node, then this particle of the contour $i$ will be in the cell $(i, j-1)$ at the moment $T+1, j=1,2, \ldots, N_{i}-1, i=1, \ldots, L$. Hence the node is ahead of the cell $(i, 0)$, and the particle, occupying the cell $(i, j)$, will be in the cell $(i, 0)$ after time $j$. Suppose the cell, located ahead a particle, is occupied, at the moment $T$. Then this particle will continue occupy the same cell at time $T+1(T=0,1,2, \ldots)$. If more than one particle attempt to move through the node simultaneously, then a conflict takes place. One of these particles wins the conflict in accordance with a given conflict resolution rule. For example, the particles wins the conflict equiprobably (egalitarian rule) or the particle with the least index wins with probability 1 (priority rule). The particle, winning conflict, moves, and particles, losing the conflict, do not move at present moment.

Denote by $H_{i}(T)$ the expectation of the total number of transitions of particles on the contour $i$ in time interval $(0, T)$. The limit

$$
v_{i}=\lim _{T \rightarrow \infty} \frac{H_{i}(T)}{T M_{i}}, i=1, \ldots, L,
$$

is called the velocity of the ith contour particles if this limit exists.

The value

$$
v=\frac{M_{1} v_{1}+\cdots+M_{L} v_{L}}{M_{1}+\cdots+M_{L}} .
$$

is called the average velocity of particles.

The velocity of particles is a random value. The considered system is a finite Markov chain (Feller, 1970), (Kemeny, \& Snell, 1976), (Borovkov, 1986). If the system comes from the state $i$ to the state $j$ after a finite time with positive probability, and the system cannot come from the state $j$ to the state $i$ after a finite time, then the state $i$ is called inessential. If a state is not inessential, then this state is called essential. If the system can come from the state $i$ to the state $j$ after a finite time, and the system can come from the state $j$ to the state $i$ after a finite time, then the states $i$ and $j$ are called communicated with each other. The set of essential states is divided into communicating classes. If there is only one communicating class, then there exist steady state probabilities, and the value of particles velocity is the same with probability 1 . Suppose there are more than one communicating class. If the initial state belongs to one of communicating classes, then the value of particles velocity depends on the class. If the initial state is inessential, then the chain comes to one of communicating class after time with a finite expectation. The chain comes, with positive probabilities, to different communicating classes, and values of velocities depend on the communicating class. Thus the limit (1) exists with probability 1, this limit is a random value, and the distribution of this value depends on the initial state of the system.

\subsection{Conditions of Synergy (Self-organization) on BAN}

The system is in the state of synergy after a moment $T$ if, after this moment, all particles move at every moment.

Suppose

$$
\left(k_{11}, \ldots, k_{1 M_{1}} ; \ldots ; k_{L 1}, \ldots, k_{L M_{L}}\right)
$$

is a vector such that $k_{i 1}, \ldots, k_{i M_{i}}, 0 \leq k_{i 1}<\cdots<k_{i M_{i}} \leq N_{i}-1$, are indexes of cells, where particles of the $i$ th contour are located, $i=1, \ldots, L$. Then this vector is called a state of the system.

Denote by $G C D\left(N_{1}, \ldots, N_{L}\right)$ the greatest common divisor of the numbers $N_{1}, \ldots, N_{L} ; G C D\left(N_{i_{1}}, N_{i_{2}}\right)$ is the $G C D-$ divisor of the numbers $N_{i_{1}}$ and $N_{i_{2}}, i_{1} \neq i_{2}, 1 \leq i_{1}, i_{2} \leq L$.

Consider the equation

$$
a x+b y+c=0,
$$

where $a$ and $b$ are any integer numbers not equal to 0 , and $c$ is any integer number. Suppose the numbers $a$ and $b$ are coprime. Indeed, if $a$ and $b$ are not coprime, then we divide both parts of (2) by the $G C D$ of the numbers $a$ and $b$. Then we obtain a similar equation, and $a, b$ are coprime. There exist integer solutions of (2) if and only if $G C D(a, b)$ is the divisor of the number c (Buchstab, 1972). Suppose that this condition is fulfilled. 
Suppose $x=x_{0}, y=y_{0}$ is an integer solution of Equation (2). Then the formulas

$$
x=x_{0}-b t, y=y_{0}+a t
$$

give all solutions of Equation (2), $t=0, \pm 1, \pm 2, \ldots$

Theorem 1 (Criterion of Synergy). A necessary and sufficient condition for the state

$$
\left(k_{11}, \ldots, k_{1, M_{1}} ; \ldots ; k_{L 1}, \ldots, k_{L, M_{L}}\right)
$$

of BAN with individual movement to be a state of synergy is that the following conditions be fulfilled

(1) $\forall l, 1 \leq l \leq L, \forall i, 1 \leq i \leq M_{l}$,

$$
k_{l, i+1}-k_{l i}>1
$$

(2) $\forall i_{1}, i_{2}, 1 \leq i_{1}, i_{2} \leq L, i_{1} \neq i_{2}, \forall j_{1}, j_{2}, 1 \leq j_{1} \leq M_{i_{1}}, 1 \leq j_{2} \leq M_{i_{2}}$,

$\operatorname{GCD}\left(N_{i_{1}}, N_{i_{2}}\right)$ is not a divisor of the number $\left(k_{i_{1} j_{1}}-k_{i_{2} j_{2}}\right)$.

Proof. If there are particles in neighboring cells of a contour, then one of these particles does not move at the present moment. Therefore the system is not in the state of synergy. Suppose there are no particles in neighboring cells on any contour. Let us show that the considered state is a state of synergy if and only if, for any $i_{1}, i_{2}, j_{1}, j_{2}\left(i_{1} \neq i_{2}, 1 \leq j_{1} \leq M_{i_{1}}\right.$, $1 \leq j_{2} \leq M_{i_{2}}$ ), there exist no nonnegative integer solutions $x, y$,

$$
N_{i_{1}} x-N_{i_{2}} y=k_{i_{1} j_{1}}-k_{i_{2} j_{2}}
$$

Indeed, suppose the system is in the state

$$
\left(k_{11}, \ldots, k_{1 M_{1}}, \ldots, k_{11}, \ldots, k_{L M_{L}}\right)
$$

If, for some $i_{1} \neq i_{2}, j_{1}, j_{2}\left(1 \leq j_{1} \leq M_{i_{1}}, 1 \leq j_{2} \leq M_{i_{2}}\right)$, there exists a solution $\left(x_{0}, y_{0}\right)$ of Equation (3), then there will be a conflict after a finite time. If for any $i_{1}, i_{2}, j_{1}, j_{2}\left(1 \leq j_{1} \leq M_{i_{1}}, 1 \leq j_{2} \leq M_{i_{2}}\right)$, there exists no integer solution of Equation (3), then there will be no delays, i,e. the system is in the state of synergy. There exists an integer solution of Equation (3) if and only if $G C D\left(N_{i_{1}}, N_{i_{2}}\right)$ is a divisor of $k_{i_{1} j_{1}}-k_{i_{2} j_{2}}$. Thus we obtain the statement of Theorem 1.

We shall formulate a necessary condition for at least one state of synergy to exist. The ratio of the particles of the contour $i$ to the number of cells of this contour is called the density of particles of the contour $i$ and is denoted by $r_{i}$,

$$
r_{i}=\frac{M_{i}}{N_{i}}, i=1, \ldots, L .
$$

Denote by

$$
A=\operatorname{LCM}\left(N_{1}, \ldots, N_{L}\right)
$$

the least common multiple of $N_{1}, \ldots, N_{L}$.

Theorem 2. Suppose movement on BAN is individual. Then a necessary condition for at least one state of synergy to exist is that (1) $\forall i, j, 1 \leq i \neq j \leq L$

$$
G C D\left(N_{i}, N_{j}\right)>1
$$

(2) $\forall i, 1 \leq i \leq L$

$$
r_{i} \leq \frac{1}{2}
$$

(3)

$$
r_{1}+r_{2}+\cdots+r_{L} \leq 1 \text {. }
$$

Proof

(1) Consider the system state

$$
\left(k_{11}, \ldots, k_{1 M_{1}} ; \ldots ; k_{L 1}, \ldots, k_{L, M_{L}}\right) .
$$

If (4) is not true, i.e., numbers $N_{i_{1}}, N_{i_{2}}$ are coprime, then there exists a solution of Equation (3) for any $k_{i_{1} j_{1}}, k_{i_{2} j_{2}}$. From this, taking into account Theorem 1, we obtain the first statement of Theorem 2. 
(2) If (5) is not true, then, at any time, the number of vacant cells of the contour is less than the number of particles. Therefore there exist particles such that these particles cannot move at present time. From this we obtain the second statement of Theorem 2.

(3) Suppose that the system is in the state of synergy. Then, in any time interval of length $A$, there are $r_{i} A$ moments such that a particles of $i$ th contour moves through the node. Since more one particle cannot move through the node simultaneously, then $r_{1} A+\cdots+r_{L} A \leq A$, i.e., (6) is true. Theorem 2 has been proved.

Theorem 3. Let $L$ be the number of contours, $N_{i}$ be the number of cells of the contour $i, M_{i}$ be the number of particles of the contour $i, i=1, \cdots, L$. Suppose the movement on BAN is individual. Then a sufficient condition for at least one state of synergy to exist is that at least one of the following conditions be fulfilled.

(1) There is only one particle on each contour, $M_{1}=\cdots=M_{L}=1$, and

$$
\operatorname{GCD}\left(N_{1}, \ldots, N_{L}\right) \geq L .
$$

(2) $L$ is divider of numbers $N_{1}, N_{2}, \ldots, N_{L}$ and $r_{i} \leq \frac{1}{L}$ for all $i=1, \ldots, L$.

Proof. (1) Suppose (7) is true and, at time $T_{0}$, the system is in the state such that the particle of the contour $i$ is in the cell $\left(i, k_{i}\right)$, and the remainder of the division of $k_{i}$ by $G C D\left(N_{1}, \ldots, N_{L}\right)$ equals $i, i=1, \ldots, L$. Such state exists in accordance with (7). Suppose a conflict of particles of contours $i$ and $j$ takes place at moment $T \geq T_{0}$. Then there exist integer non-negative solutions $x=x_{0}, y=x_{0}$ of the equation

$$
N_{i} x-N_{j} y=k_{j}-k_{i}
$$

If such solution $x_{0}, y_{0}$ exists, then $G C D\left(N_{i}, N_{j}\right)$ is a divisor of $k_{j}-k_{i}$, and therefore $G C D\left(N_{1}, \ldots, N_{k}\right)$ is also a divisor of $k_{j}-k_{i}$. However the remainders of the division of numbers $k_{i}$ and $k_{j}$ by $G C D\left(N_{1}, \ldots, N_{L}\right)$ are not equal to each other. Therefore $G C D\left(N_{1}, \ldots, N_{k}\right)$ is not a divisor of $k_{i}-k_{j}$. This contradiction proves the first statement of the theorem.

(2) Suppose $L$ is a divisor of the numbers $N_{1}, N_{2}, \ldots, N_{L}$, and $r_{i} \leq \frac{1}{L}$ for all $i=1, \ldots, L$. Assume that particles of the contour $i$ are in cells such that remainders of the division of cells indexes by $L$ are equal to $i(i=1, \ldots, L)$. We shall prove that this state is a state of synergy. Suppose a conflict of the contours $i$ and $j$ takes place at the moment $T \geq T_{0}$. Then there exist integer non-negative solutions $x=x_{0}, y=x_{0}$ of the equation

$$
N_{i} x-N_{j} y=k_{j}-k_{i}
$$

If such solution $x_{0}, y_{0}$ exists, then $G C D\left(N_{i}, N_{j}\right)$ is a divisor of $k_{i}-k_{j}$. Since $L$ is a divisor of $N_{i}$ and $N_{j}$, we see that $L$ is also a divisor of $k_{j}-k_{i}$. However remainders of the division of $k_{i}, k_{j}$ by $L$ are not equal to each other. This contradiction proves the second statement of the theorem. Theorem 3 has been proved.

Remark 1. Parameters of BAN with individual movement can be chosen such that both necessary conditions of Theorem 2 are fulfilled, and there is no state of synergy.

We give an example. Suppose $L=2, N_{1}=6, N_{2}=3, M_{1}=3, M_{2}=1$. Then we have $r_{1}+r_{2}=\frac{M_{1}}{N_{1}}+\frac{M_{2}}{N_{2}}=\frac{5}{6}<1$, i.e. both conditions of Theorem 2 are fulfilled. However we shall prove that there exists no state of synergy. Without loss of generality, we assume that the cell $(1,1)$ is occupied. If $\left(k_{11}, k_{12}, k_{13} ; k_{2}\right)$ is the state of synergy, then $k_{11}=1, k_{12}=3$, $k_{13}=5$. In accordance of Theorem 1, in the case of state of synergy, $\operatorname{GCD}(3,6)=3$ is not a divisor of $k_{11}-k_{2}, k_{12}-k_{2}$, $k_{13}-k_{2}$. However this condition is not fulfilled for none of states $(1,3,5 ; 0),(1,3,5 ; 1),(1,3,5 ; 2)$. Thus there is no state of synergy.

\section{BAN with Contours of the Same Length Necessary and Sufficient Condition for Self-organization of Individual Movement}

Let $L$ is the number of contours, $N_{i}$ is the number of cells of the contour $i, M_{i}$ is the number of particles of the contour $i$, $i=1, \cdots, L$. Assume that the number of cells on each contour is the same, $N_{1}=\cdots=N_{L}=N$.

Theorem 4. Let movement be individual. A necessary and sufficient condition for at least one state of synergy to exist is that

(1) $\forall i, 1 \leq i \leq L$

$$
r_{i} \leq \frac{1}{2}
$$

$$
M_{1}+M_{2}+\cdots+M_{L} \leq N
$$


Proof. In accordance with statements 2, 3 of Theorem 2, we have that the condition is necessary.

Let us show that the condition is sufficient. Assume that $M_{1} \geq M_{i}, i=2, \ldots, L$. Put particles of the contour 1 into cells with indexes $0,2,4, \ldots, 2\left(M_{1}-1\right)$, put particles of the contour 2 into cells with indexes $2 M_{1}, 2 M_{1}+2, \ldots, 2\left(M_{1}+M_{2}\right)-1$, etc. If all cells with even indexes are already occupied, then we put remaining particles into cells with odd indexes. In accordance with the second condition of the theorem all particles will be located in cells with different indexes, and there will be no particles in neighboring cells of any contour. Thus there exists a state of synergy. Theorem 4 has been proved.

\section{Total-connected Movement on BAN}

Let $L$ be the number of contours, $N_{i}$ be the number of cells of the contour $i, M_{i}$ be the number of particles of the contour $i, i=1, \cdots, L$.

\subsection{Synergy of BAN with total-connected movement}

Theorem 5. Let the movement be total-connected. Then a state $\left(k_{11}, \ldots, k_{1 M_{1}}, \ldots, k_{L 1}, \ldots, k_{L M_{L}}\right)$ is a state of synergy if and only if, $\forall i_{1}, i_{2}, j_{1}, j_{2}\left(i_{1} \neq i_{2}, 1 \leq j_{1} \leq M_{i_{1}}, 1 \leq j_{2} \leq M_{i_{2}}\right), \operatorname{GCT}\left(N_{i_{1}}, N_{i_{2}}\right)$ is not a divisor of $\left(k_{i_{1} j_{1}}-k_{i_{2} j_{2}}\right)$.

The proof of Theorem 5 is similar to the proof of Theorem 1 .

Suppose there is an ordered set of clusters on each contour. There are $l_{i}$ clusters on the contour $i$, and the sth cluster contains $M_{i s}$ particles, $s=1, \ldots, l_{i}, M_{i 1}+\cdots+M_{i, l_{i}}=M_{i}, M_{i}<N_{i}, i=1, \ldots, L$.

Theorem 6. Let the movement be total-connected. Then a necessary condition for at least one state of synergy to exist is that

(2) $\forall i, j \neq\{1, \ldots, L\}$

$$
r_{1}+\cdots+r_{L} \leq 1
$$

$$
\max \left(M_{i 1}, \ldots, M_{i N_{i}}\right)+\max \left(M_{j 1}, \ldots, M_{j N_{j}}\right) \leq G C D\left(N_{i}, N_{j}\right)
$$

The proof of the first statement of Theorem 6 is similar to the proof of the third statement of Theorem 2.

In accordance with Theorem 5, for the state of synergy, the sum of two clusters of different contours cannot be greater than $G C D\left(N_{i}, N_{j}\right)$. From this, the second statement follows. Theorem 6 has been proved.

Theorem 7. Let the movement be total-connected. Then a sufficient condition for at least one state of synergy to exist is that

$$
M_{1}+\cdots+M_{L} \leq G C D\left(N_{1}, \ldots, N_{L}\right)
$$

The proof of Theorem 7 is similar to proof of the first statement of Theorem 3.

The behavior of BAN depends on the initial state and on the realization of the process if the initial state is fixed. Let us give examples.

In the following example, the initial state is fixed, but, with positive probabilities, the velocities and limit numbers of clusters on contours are different, and either the system behavior will be deterministic after a time interval with finite expectation or the system will come to a set of states such that deterministic behavior is not possible.

Suppose $L=2, N_{1}=7, N_{2}=3, M_{1}=2, M_{2}=1$. Each particle of the contour 1 wins every conflict with probability $\frac{2}{3}$. Assume that the initial state is $(0,5 ; 0)$. There is a conflict in this state. If the particle of the contour 1 wins the conflict, then particles of this contour form a cluster, the behavior of system will be deterministic, and the following sequence of states repeats periodically

$$
\begin{aligned}
& (5,6 ; 0) \rightarrow(4,5 ; 1) \rightarrow(3,4 ; 0) \rightarrow(2,3 ; 1) \rightarrow \\
\rightarrow & (1,2 ; 0) \rightarrow(0,1 ; 1) \rightarrow(6,1 ; 0) \rightarrow(5,6 ; 0) \ldots
\end{aligned}
$$

The values of velocities are

$$
v_{1}=1, v_{2}=\frac{6}{7}, v=\frac{2 v_{1}+v_{2}}{3}=\frac{20}{21}
$$

If the particle of the contour 2 wins the conflict in state $(0,5 ; 0)$, then particles of this contour do not form a cluster, the behavior of system will never be deterministic, and, with probability 1 , the values of velocities are

$$
v_{1}=\frac{35}{36}, v_{2}=\frac{8}{9}, v=\frac{17}{18} \text {. }
$$


The following example shows that, in the case of total-connected movement on contours of the same length, it is possible that the system will not come to the state of synergy, and the limit number of clusters on a contour is not equal to 1.

Suppose $N_{1}=N_{2}=25, M_{1}=M_{2}=13$ and the initial state is

$$
(1,2,3,4,10,11,12,13,14,18,19,20,21 ; 4,5,6,7,8,9,15,16,17,22,23 \text {, }
$$

$$
\text { 24, 25). }
$$

Then we have deterministic movement with period 26. Velocities of particles are

$$
v_{1}=v_{2}=\frac{25}{26} \text {. }
$$

The limit number of clusters on each contour equals 3.

\subsection{Synergy and Velocity of Total-connected Movement on BAN with Contours of the Same Length}

Suppose that the length of each contour is the same, $N_{1}=\cdots=N_{L}=N$. We give the definition of a group of clusters. A set of clusters (these clusters can be located on different contours) is called a group of clusters if the set of indexes of cells, where these clusters are located, is a connected set on the integer numbers segment $[0, \ldots, N-1]$. Group of the maximum length is called a maximum group (maximum cluster). If clusters of a maximum group do not contain any cells with the same numbers, then this group is called a maximum simple group (maximum simple cluster). It is evident that the length of the supporter of a maximum simple group is equal to the sum of lengths of all clusters such that these clusters are contained in the maximum group.

Suppose values $L, M_{1}, \ldots, M_{L}, N$ are given. We formulate the necessary and sufficient condition of synergy.

Theorem 8. A necessary and sufficient condition for BAN with total-connected movement to come to the state of synergy after a time with a finite expectation is that

$$
M_{1}+\cdots+M_{L} \leq N
$$

Lemma 1. Suppose the movement is total-connected and $N_{1}=\cdots=N_{L}$. Then any maximum group cannot become thinner, i.e., this group cannot lose any clusters or their particles.

Proof. Suppose clusters of a maximum group $G$ do not hinder the movement of clusters, not contained in the maximum group, and clusters, not contained in the maximum group, do not hinder the movement of clusters of $G$. Then the set of clusters of the group does not change. Suppose a cluster $C$ does not belong to the group $G$, and this cluster delays the movement of clusters of $G$, or clusters of $G$ delays the movement of the cluster $C$. Then a group is formed such that this group contains both the clusters of the group $G$ and the cluster $C$. In all these cases, the maximum group does not lose both clusters and particles. Lemma 1 has been proved.

Lemma 2. Let the movement be total-connected. Suppose $N_{1}=\cdots=N_{L}$. At any moment, the set of clusters on BAN is a union of nonself-intersecting maximum clusters.

The statement of Lemma 2 is obvious.

Lemma 3. Assume that the movement is total-connected and $N_{1}=\cdots=N_{L}=N$. Suppose the velocity and configuration of a maximum cluster do not change during $N$ time units (indexes of cells, which are occupied by the cluster, shift onto 1 per time unit). Then this maximum cluster is simple.

Proof. Lemma 3 follows from the definition.

Lemma 4. Let the movement be total-connected. Suppose $N_{1}=\cdots=N_{L}=N$, the system is not in the state of synergy and there is no maximum cluster, containing more than $N-1$ particles. Then, for time interval of duration $N$, either some maximum clusters merge or supporters of some clusters, which are not simple, increase.

Proof. Suppose $N_{1}=\cdots=N_{L}=N$, the system is not in the state of synergy and there is no maximum cluster, containing more than $N-1$ particles. If no cluster is delayed during $N$ time units, then a sequence of $N$ states will repeat periodically. If a delay takes place, then some groups, which are not simple, increase, and it is possible that some maximum clusters merge. Lemma 4 has been proved.

Lemma 5. Suppose the movement is total-connected and $N_{1}=\cdots=N_{L}=N$. If inequality (8) is fulfilled, then, for all $T_{1}$, there exists a moment $T_{2}$ such that no particle moves through the node at this moment, and $T_{1} \leq T_{2}<T_{1}+N$.

Proof. Any particle cannot move through the node more than one time during a time interval of duration $N$. Since the number of particles is less than $N$, we obtain Lemma 5. 
Lemma 6. Suppose $N_{1}=\cdots=N_{L}=N$, the movement is total-connected and (8) is true. Then all clusters will be maximum simple clusters after a finite time. We have the process of movement without conflicts, with minimum configuration, and maximum velocity.

Proof Suppose (8) is true. Then, in accordance with Lemma 4, no delays of clusters will be in future or a maximum cluster increases. However maximum cluster cannot increase more than a finite times. From this, Lemma 6 follows.

Proof of Theorem 8 . The condition is necessary in accordance with Theorem 6. Let us show that the condition is sufficient. In accordance with (8) and Lemma 4, after a finite moment, any cluster will be in a maximum group such that clusters of other groups (if there are other groups) do not hinder clusters of the group. Theorem 8 has been proved.

Suppose

$$
M_{1}+\cdots+M_{L}>N
$$

Lemma 7. Suppose $N_{1}=\cdots=N_{L}=N$, the movement is total-connected and (9) is true. Then there exists a moment $T_{0}$ such that after this moment just one particle moves through the node.

Proof. Assume that $N_{1}=\cdots=N_{L}=N$. Suppose there is a delay of a maximum group $G$ cluster at the moment $T_{2}<T_{1}+N$. Then either a group of greater length forms, and this group contains all clusters of the group $G$, or, there exists a moment $T_{0}$ such that, for all $j \in\{0,1, \ldots, N-1\}$, the cell $j$ is occupied at least on one contour. Hence, $\forall T_{1}>T_{0}$ the cell $j$ is occupied at least one contour, $j \in\{0,1, \ldots, N-1\}$. Indeed, assume that it is not true. Then the cell $j+1$ (addition modulo $N$ ) of any contour was not occupied at the preceding moment. At least one particle moves through the node at each moment after the moment $T_{0}$. However more than one particle cannot move through the node simultaneously. Lemma 6 has been proved.

Suppose (9) is true. Let us consider the system behavior after moment $T_{0}$ such that this moment satisfies condition of Lemma 7.

Lemma 8. Let the movement be total-connected. Assume that $N_{1}=\cdots=N_{L}=N$. Suppose a particle P is the leader of a cluster, and, at moment $T_{1}>T_{0}$, this particle moves through the node to the cell $N-1$ of the contour. Then this particle moves at the moments $T_{1}+1 \ldots, T_{1}+N-1$ and the particle will be in the cell before the node (cell 0$)$ at the moment $T_{1}+N-1$.

Proof. Since a sequence of system states repeats periodically after the moment $T_{0}$, we see that clusters cannot merge and form clusters of greater length. Therefore a cluster cannot be delayed unless the leader of the cluster is before the node, and the cluster moves $N$ time units after crossing the node. From this, Lemma 8 follows.

Lemma 9. Assume that $N_{1}=\cdots=N_{L}=N$. Suppose the movement is total-connected, and a particle P moves through the node at time moments $T_{1}$ and $T_{2}, T_{0}<T_{1}<T_{2}$. Then $T_{2}-T_{1} \geq M_{1}+\cdots+M_{L}$.

Proof. The particle $P$ is located in the cell $N-1$ at moment $T_{1}$. No other particle can be in the cell $N-1$ of the contour. Indeed, if a particle occupies the cell $N-1$ at the moment $T_{1}$, then, in accordance with Lemma 8, this particle moves through the node at this moment. However two particles cannot move through the node simultaneously. Therefore it is true the following. Suppose a particle is not the particle $P$. Then, at the moment $T_{1}$, either this particle is in the queue before the node or, in accordance with Lemma 8 , this particle comes to the node earlier than the particle $P$. Therefore any particle (not the particle $P$ ) moves through the node between moments $T_{1}$ and $T_{2}$. From this, taking into account that more one particle cannot move through the node simultaneously, we obtain Lemma 9.

Lemma 10. Assume that $N_{1}=\cdots=N_{L}=N$. Let the movement be total-connected. Suppose a particle $P$ and moments $T_{1}, T_{2}$ satisfy Lemma 9. Then the equality $T_{2}-T_{1}=M_{1}+\cdots+M_{L}$ is true.

Proof. From Lemma 9, it follows that the inequality $T_{2}-T_{1} \geq M_{1}+\cdots+M_{L}$ is true.

Since just one particle moves through the node at each moment, we have see that the inequality $T_{2}-T_{1}>M_{1}+\cdots+M_{L}$ can be true only if at least one particle crosses the node more than one time during the time segment $\left[T_{1}, T_{2}\right]$. However this is impossible. Suppose a particle moves through the node in this time segment. Then, in accordance with Lemma 8 , this particle returns to the node later than the particle $P$, and therefore the priority of the considered particle is lower than priority of the particle $P$. This contradiction proves Lemma 10.

Lemma 11. Assume that $N_{1}=\cdots=N_{L}=N$. Let the movement be total-connected. Suppose (9) is true. Then time intervals of particle movement and waiting alternate. Any particle moves for $N$ time units and waits for $M_{1}+\cdots+M_{L}-N$ times units.

Proof. If a particle is a leader of a cluster, then Lemma 11 is true for this particle in accordance with Lemmas 8,10 . Since all particles of any cluster move simultaneously, we obtain Lemma 11. 
Theorem 9. Assume that $N_{1}=\cdots=N_{L}=N$. Suppose the movement is total-connected. Then velocities of particles on all contours are the same, and

$$
v_{1}=\cdots=v_{L}=\left\{\begin{array}{l}
1, M_{1}+\cdots+M_{L} \leq N, \\
\frac{N}{M_{1}+\cdots+M_{L}}, M_{1}+\cdots+M_{L}>N .
\end{array}\right.
$$

Proof. If (8) is true, then synergy takes place. Therefore,

$$
v_{1}=\cdots=v_{L}=1 .
$$

If (9) is true, then, in accordance of Lemma 10,

$$
v_{1}=\cdots=v_{L}=\frac{N}{M_{1}+\cdots+M_{L}} .
$$

Theorem 9 has been proved.

Corollary 1. Assume that $N_{1}=\cdots=N_{L}$ and the movement is total-connected. Let values of $L, N, M_{1}, \ldots, M_{L}$ be given. Then velocities of particles on any contour do not depend on the initial state of the system.

\section{Total-connected Movement on BAN with One Cluster on Every Contour}

\subsection{Synergy of Two Clusters}

Assume that the number of contours equals 2, and there is only one cluster on each contour. We formulate a necessary and sufficient condition of synergy.

Theorem 10. Suppose $L=2$, and there is a cluster on each contour. Then a necessary and sufficient condition for the system to come to the state of synergy after a finite time is that

$$
M_{1}+M_{2} \leq G C D\left(N_{1}, N_{2}\right)
$$

Proof. Suppose the system is in the state such that $\left(k_{1}, k_{2}\right)$ are coordinates of leaders clusters, $\left(0 \leq k_{1} \leq N_{1}-1\right.$, $\left.0 \leq k_{2} \leq N_{2}-1\right)$. If (10) is not true, then $\operatorname{GCD}\left(N_{1}, N_{2}\right)$ is a divisor of numbers $M_{1}+2, \ldots, k_{2}-k_{1}, \ldots, k_{2}-k_{1}+M_{2}-2$, $k_{2}-k_{1}+M_{2}-1$. Taking into account Theorem 7, we see that the condition is sufficient. If (10) is true, then either the system is in the state of synergy, or a delay of a cluster takes place after a finite time. This cluster begins to move again when the system is in the state $\left(0, N_{2}-M_{2}\right)$ or $\left(N_{1}-M_{1}, 0\right)$. From (10), it follows that these states satisfy the condition of synergy (Theorem 5). From this, Theorem 10 follows.

\subsection{Velocity of Particles on BAN with Two Contours and a Cluster on Each Contour}

Assume that there are two contours and a cluster on each contour. We shall describe an approach to velocities calculation. Suppose the condition of synergy (10) is not fulfilled. Denote by $A$ a set of system states such that a state belongs to this set if and only if one of clusters do not moves in this state. After being in the set $A$, the system comes either into the state $\left(0, N_{2}-M_{2}\right)$, if there was a delay of the cluster 1 , or into the state $\left(N_{1}-M_{1}, 0\right)$, if there was a delay of the cluster 2 . For each of these two states, analyzing the appropriate linear equation with two variables, we can calculate after what time interval the system comes to a state of the set $A$ and to what state of this set $A$ the system comes. Taking into account results of this analysis, we can calculate velocities $v_{1}, v_{2}$. Depending on values of system parameters, there is a pair of values $\left(v_{1}, v_{2}\right)$ such that these values are taken with probability 1 , or there are two pairs of values $\left(v_{1}, v_{2}\right)$ such that these values are taken with positive probabilities. In the latter case, the velocities depend on the initial state, and there exist initial states such that each pair of values is taken with positive probability.

\section{Comments, and Further Research}

(6.1) We have found a necessary and sufficient condition of synergy and formulas for velocities in the case of contours of the same length and in the case of two contours of any length. We have found some necessary conditions and some sufficient conditions of synergy on a flower with contours of different lengths. In general case, problems of formulation of necessary and sufficient condition of synergy and calculation of velocities, in cases of total-connected or individual condition have not been solved.

(6.2) For analysis of general characteristics of the behavior of dynamical systems on networks, it is interesting to investigate systems with regular structures such that it is possible to find analytical characteristics of their behavior.

(6.3) An alternating flower (BAN) is considered in the present paper. Some results can be reformulated for the flower with a common cell, which is also the common node. The condition of synergy (Theorem 1) changes. This condition takes 
into account that a delay of one particles takes place not only if particles come to the node simultaneously, but also if one of the particles comes at next moment. In accordance with this, the inequality $\operatorname{GCD}\left(N_{i}, N_{j}\right)>3$ substitutes (4), and the inequality

$$
r_{1}+r_{2}+\cdots+r_{L} \leq \frac{1}{2}
$$

substitutes (6).

(6.4) We intend to investigate generalizations of the system, considered in the present paper, e.g., the system with several neighboring (Figure 3) or equidistant from each other joined nodes and cells.

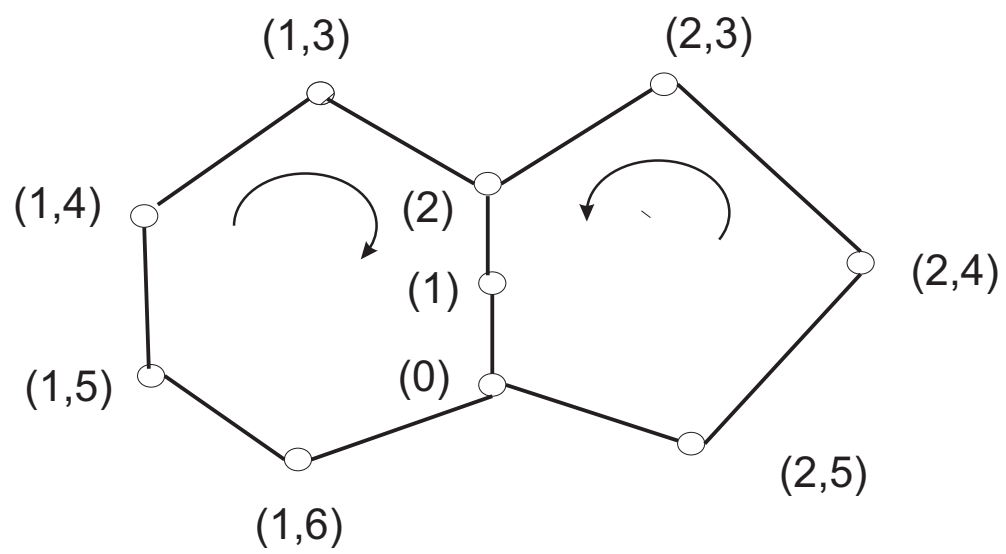

Figure 3. Contours with three common cells joined with nodes

(6.5) There exist initial states such that particles velocities (in general case, probability distribution of velocities) are different.

(6.6) As we mentioned in Section 1, in [1] - [2], a dynamical system has been introduced. In this system, clusters move on a "flower" ("bouquet"). The state space and time scale of this system are continuous. We can reformulate results of the present paper for this continuous system. For example, consider a bouquet with two closed contours. They are the contour 1 of length $L_{1}$, and the contour 2 with length $L_{2}$, where $L_{1}$ and $L_{2}$ are positive numbers. The cluster of length $l_{i}$ $\left(l_{i}<L_{i}\right)$ moves on the cluster $i, i=1,2$. The point $(0,0)$ is the node, i.e., common point of these contours. Each state of the system is $\left(x_{1}, x_{2}\right)$, where $x_{i}$ is the rear point of the cluster, $0 \leq x_{i}<L_{i}, i=1,2$. Two clusters cannot move through the node simultaneously. In the state $(0,0)$, a conflict of clusters takes place. Denote by $T_{i}$ the duration of time interval such that, during this time interval, the cluster $i$ passes the distance equal to the contour length if there are no delays: $T_{i}=l_{i} / v_{i}$. Consider a time interval such that, during this time interval, the cluster $i$ passes through the node. The duration of this interval is equal to $l_{i} / v_{i}, i=1,2$. If the ratio $T_{1} / T_{2}$ is an irrational number, then the system cannot come to the state of synergy. Suppose that the ratio $T_{1} / T_{2}$ is a rational number. Denote by $G C D\left(T_{1}, T_{2}\right)$ the greatest divisor of $T_{1}$ and $T_{2}$. If $T_{1} / T_{2}$ is rational, then a necessary and sufficient condition for the system to come to the system of synergy after a finite time is that

$$
\frac{l_{1}}{v_{1}}+\frac{l_{2}}{v_{2}} \leq G C D\left(T_{1}, T_{2}\right)
$$

(11) is similar to (10).

\section{References}

Blank, M. (2000). Exact analysis of dynamic systems appearing in traffic flows models. Uspekhi matematicheskikh nauk, 55(3), $167-168$.

Borovkov, A. A. (1986). Probability theory (2th ed.) Moscow: Nauka. (In Russian.)

Buchstab, A. A. (1972). Number theory. Moscow, Nauka. (In Russian.)

Bugaev, A. S., Buslaev, A. P., Kozlov, V. V., Tatashev, A. G., \& Yashina, M. V. (2013). Traffic modeling: Monotonic random walks on network. Matematicheskoye modelirovaniye, 25(8), $3-21$.

Buslaev, A. P. (2010). Traffic flower with $n$ petals Journal of Applied Functional Analysis (JAFA) 5(1), 85 - 99.

Buslaev, A.P., \& Tatashev, A. G. (2016). Bernoulli algebra on common fractions and generalized oscillations Journal of Mathematics Research, 8(3), 82 - 93. http://dx.doi.org/105539/jmr.v8n3p82 
Buslaev, A. P., \& Tatashev, A. G. (2015). Generalized real numbers pendulums and transport logistic applications. In New Developments in Pure and Applied Mathematics, 388 - 392. Vienna. www.inase.org/library/2015/vienna/bypaper/MAPUR/MAPUR-63.pdf

Buslaev, A. P., \& Yashina, M. V. (2009). About flows on a traffic flower with control. MCV. The World Congress in Computer Science, Computer Engineering, and Applied Computing (WORLDCOMP'09), LAS Vegas, Nevada USA (July 13 - 16, 2009) in Proc. of the 2009 International Conference on Modelling Simulation and Visualization, CSREAS Press, $254-257$.

Feller, W. (1970). An introduction to probability theory and its applications, 1. New York, John Willey.

Halmos, P. R. Lectures on Ergodic Theory. Publ. Math. Soc. Japan, Tokyo, 1956; reprinted Chelsea, New York, 1960.

Kemeny, J. G., \& Snell, J. L. (1976). Finite Markov chains. New York, Heidelberg, Tokyo: Springer Verlag.

Kozlov, V. V., Buslaev, A. P., \& Tatashev, A. G. (2014). Behavior of pendulums on a regular polygon. Journal of Communication and Computer, 11, $30-38$.

Kozlov, V. V., Buslaev, A. P., Tatashev, A.G., \& Yashina, M. V. (2014). Monotonic walks of particles on a chainmail and coloured matrices. Proceedings of the 14th International Conference on Computational and Mathematical Methods in Science and Engineering, CMSSE 2014, Cadiz Spain, June 3 - 7 2014, 3, 801 - 805.

Kozlov, V. V., Buslaev, A. P., \& Tatashev, A. G. (2015). Monotonic walks on a necklace and coloured dynamic vector. International Journal of Computer Mathematics, 92(9), 1910 - 1920. http://dx.doi.org/1080/00207160.2014/915964

Kozlov, V. V., Buslaev, A. P., Tatashev, A. G., Yashina, M. V. (2015). Dynamical systems on honeycombs. Traffic and Granular Flow' '13. Springer - Verlag Heidelberg, $441-452$.

Nagel, K., \& Schreckenberg, M. (1992). A cellular automation models for freeway traffic. J. Phys. I. France, 2, 2221 2229.

Schadschneider, A., Schreckenberg, M. (1993). Cellular automation models and traffic flow. J. Phys. A. Math. Gen., 51, L679 - L683.

\section{Copyrights}

Copyright for this article is retained by the author(s), with first publication rights granted to the journal.

This is an open-access article distributed under the terms and conditions of the Creative Commons Attribution license (http://creativecommons.org/licenses/by/4.0/). 